\title{
Mathematical modeling of Pickering emulsions stabilization process by solid nanoparticles
}

\author{
Yaroslavna Kashyrina, Oleksii Muratov, \\ Georgii Sokolskyi, Oleg Miroshnikov
}

\author{
National University of Food Technologies, Kyiv, Ukraine
}

Keywords:

Pickering

Nanoparticles

Silica

Carbon

Modeling

Article history:

Received 29.04.2017

Received in revised form 15.06.2017

Accepted 05.09.2017

Corresponding author:

Oleksii Muratov

E-mail:

alzr@nuft.edu.ua

DOI: $10.24263 / 2304-$

974X-2017-6-3-12

\section{Abstract}

Introduction. The aim of this work is to predict and study the conditions for the stabilization of food and cosmetic emulsions with the Pickering effect establishing the possibility of mathematical modeling of conditions for the most effective stabilization of emulsions.

Materials and methods. It was studied a model emulsions with injected solid nanoparticles based on water and triglycerides of carboxylic acids such as Glyceryl tributyrate (C4), Glyceryl tricaprylate (C6), and Glyceryl trioleate, linseed oil (a mixture of triglycerides of acids: 9$11 \%$ of palmitic and stearic, $13-29 \%$ of oleic, $15-30 \%$ of linoleic, 44-61\% of linolenic acids) and their ethyl esters with a similar composition, and perfume oil. Calculations of surface tension and stability for the model systems of food and cosmetic emulsions were carried out by means of MathCAD-2000 software by Binks method.

Results and discussion. It was calculated free energy values of a spherical nanoparticle (in $\mathrm{k}_{\mathrm{B}} \mathrm{T}$ units, $\mathrm{T}=$ $298 \mathrm{~K}$ ) which occupies position at the water / oil interface (tributyrin, tricaprylin, triolein, linseed oil and their ethyl esters, perfume oil). The stability of a particle is characterized by the energy barrier, from the side of one of the liquids. In connection with the fact that the energy wells are parabolas, it was collected information on the magnitude of the barriers and the position of the minimum when the particle is moved from the minimum with the $z_{m}$ coordinate into water $(z=-1)$ and into oil $(z=1)$. The best energy well parameters of this solid emulsifier were about 2830 (in linseed oil and in their ethers), 4010 (perfume oil), 400 (triolein) and $150 \mathrm{k}_{\mathrm{B}} \mathrm{T}$ (tributyrin). It was shown that a nanoparticle consisting of $50 \%$ hydrophilic silica possesses the best stabilizing properties.

Conclusions. The principal possibility of theoretical prediction of the conditions for the stabilization of food and cosmetic emulsions by the Pickering effect is shown. Stabilizing particles, which corresponds to optimal selection of the nanoparticle material, are consisting of $50 \%$ hydrophilic silica. 


\section{Introduction}

Stabilization of emulsions by means of solid particles has been known for almost a century (so-called Pickering emulsion) [1], but the idea of improving the stability of emulsions by developments in nanotechnology has arisen only recently [2]. Emulsions are heterogeneous systems consisting of mutually insoluble, finely dispersed liquids. In the food, pharmaceutical and cosmetic industries emulsions are intended for internal, external or parenteral use.

Solid nanoparticles of insoluble substances are a special class of stabilizers for disperse systems, primarily emulsions and foams. Solid stabilizers are clay, coal, silica, glass, oxides, hydroxides and insoluble salts of many metals [3].

The problem of increasing the shelf life of emulsions requires the involvement of new knowledge and approaches, including mathematical modeling of physicochemical processes. Experimental confirmation of the Pickering stabilization's processes of emulsions is a rather complicated procedure, which also indicates to the necessity of conducting of model calculations for the searching the optimal material of a solid emulsifier.

Thus it was chosen food and cosmetic emulsions model with injected solid nanoparticles of different substances as the object of the study, which are already used as food additives (it has record in Codex Alimentarius). The subject of the study is the conditions for the Pickering stabilization of emulsions by solid nanoparticles.

The aim of this work is to predict and study the conditions for the stabilization of food and cosmetic emulsions with the Pickering effect establishing the possibility of mathematical modeling of conditions for the most effective stabilization of emulsions. Hence it was put such tasks in this work:

- to investigate the literature data about promising disperse food and cosmetic objects, nanodisperse solid materials for the Pickering stabilization effect;

- $\quad$ to choose the necessary techniques for theoretical study of the Pickering effect and mathematical modelling of the stabilization process;

- $\quad$ to compile a model of surface energy calculations of the stabilizing nanoparticle by means of MathCAD-2000 software and to make the necessary calculations;

- to make conclusions regarding to the optimal choice of stabilizing food additive depending of the emulsion's type.

\section{Materials and methods}

Objects of our study were model food and cosmetic emulsions with injected solid nanoparticles of various substances, which have the ability to Pickering stabilization and are used as food additives, are emulsions based on water and triglycerides of carboxylic acids such as Glyceryl tributyrate $\left(\mathrm{C}_{4}\right)$, Glyceryl tricaprylate $\left(\mathrm{C}_{6}\right)$, and Glyceryl trioleate, linseed oil (a mixture of triglycerides of acids: $9-11 \%$ of palmitic and stearic, $13-29 \%$ of oleic, $15-30 \%$ of linoleic, $44-61 \%$ of linolenic acid [4]) and their ethyl esters with a similar composition (trade name - Linaetholum), and perfume oil [5]. Subjects of our study were investigation of stabilizing effects of nanoparticles in above mentioned emulsions and ways for its theoretical prediction.

Perfume oil by chemical composition is a mixture of hydrocarbons, which are produced by deep purification of spindle oil obtained during petroleum distillation. It is colorless transparent liquid without odor and taste with a density of $0.875-0.880 \mathrm{~g} / \mathrm{cm}^{3}$. It 
boils at a temperature of about $360{ }^{\circ} \mathrm{C}$, insoluble in water and alcohol, but soluble in all organic solvents, easily fuses with waxes and fats, immiscible and stable against air, alkalis and acids. It is widely used in cosmetics for the manufacture of Vaseline [6].

Solid stabilizing nanoparticles are carbon (E153), paraffin (E905x) and silica (E551) with varying degrees of hydrophilicity.

Further in our work, "liquid 1" corresponds to triglycerides of carboxylic acids $\mathrm{C}_{4}, \mathrm{C}_{6}$ or oleic acid, or triglycerides or ethyl esters of fatty acids of linseed oil or perfume oil; "Liquid 2" is water; "Solid phase" (particle) - $\mathrm{SiO}_{2}$, paraffin or carbon. The spherical particles with a radius of $12 \mathrm{~nm}$, which corresponds to the average particle size in the AEROSIL ${ }^{\circledR} 200$ product (Evonik Industries AG) were considered.

The procedure of calculating of the energy of nanoparticles, which are located on the surface between two liquids, are given in ref. [2]. The unknown values of the surface tension values were calculated in accordance with the procedure shown in [7]. Calculations of surface tension at the surface of two phases for the model systems of food and cosmetic emulsions, which used in the work, were carried out by means of the software MathCAD2000 [6] according ref. [2].

\section{Results and discussion}

When someone perform free energy calculations of a system consisting of a particle placed on the surface between two liquids which formed an emulsion, it is necessary to distinguish between large and small particles. In the case of significant weight of the particles, the stabilizing properties of the particle are reduced due to action of external forces which move out particle from liquid-liquid interface where it was stabilized. For small nanoparticles this effect can be neglected. All kinds of interactions between stabilizing nanoparticles are also neglecting for simplicity. The double electric layer at the solid nanoparticle-liquid interface in this model is also not taken into account.

The stable position of the solid spherical particle at the interface of two phases is determined by the equilibrium edge angle $\theta_{o w}$ (Figure 1). To estimate the efficiency of the binding energy, it is usually compared with the kinetic energy of the Brownian motion ( $3 k_{B} T / 2$, where $k_{B}$ - the Boltzmann constant, the temperature $T$ in Kelvin). Calculations of free energy will be carried out in temperature units according to equations 1-2.

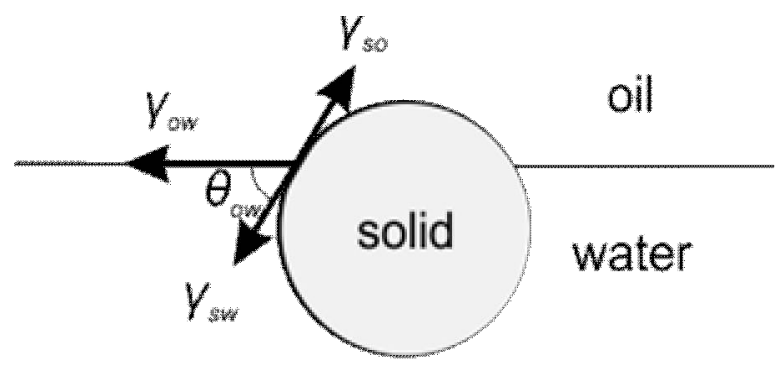

Figure 1. Schematic representation of a solid particle at the interface of emulsion phases [2]. 


$$
\begin{gathered}
\text { Food Technology - } \\
z_{0}=\frac{z}{R} ; S_{T}=4 \pi R^{2} ; A_{T}=\pi R^{2} \\
E_{P 1}=\gamma_{P 1} S_{T} \frac{\left(1+z_{0}\right)}{2} ; E_{P 2}=\gamma_{P 2} S_{T} \frac{\left(1+z_{0}\right)}{2} \\
E_{0}=\frac{E_{P 1}+E_{P 2}+E_{12}}{k_{B} T}=\frac{\pi R^{2} \gamma_{12}}{k_{B} T}\left(z_{0}^{2}+\frac{2\left(\gamma_{P 1}-\gamma_{P 2}\right)}{\gamma_{12}} z_{0}+\frac{2\left(\gamma_{P 1}+\gamma_{P 2}\right)}{\gamma_{12}}\right)
\end{gathered}
$$

where $R$ is the radius of the nanoparticle, $z$ is the distance from the interface of the liquids to the center of the particle, $z_{0}=z / R$ is the dimensionless distance, $\sigma_{\mathrm{P} 1}, \sigma_{\mathrm{P} 2}$ and $\sigma_{12}$ are a surface tension between solid particle-liquid 1, particle-liquid 2 and liquid 1-liquid 2, respectively.

In order to calculate the free energy of solid particle shown in Figure 1, it is necessary to know the interfacial tension between solid particle-water and solid particle-oil $\left(\sigma_{\mathrm{P} 2}\right.$ and $\sigma_{\mathrm{P} 1}$, respectively). These values are unknown from the experiments, but they can be calculated if the surface tension of water, oil and solid particles at the boundary with air is known. In this case, this surface tension is represented as the sum of dispersed and polar components $\left(\gamma^{\mathrm{d}}\right.$ and $\gamma^{\mathrm{p}}$, respectively). For water, the polar and dispersed parts are taken from the literature [2]. The formulas $4-7$, by which the surface tension values were calculated, can be written as follow:

$$
\begin{gathered}
\gamma_{S O}-\gamma_{S W}=\gamma_{O W} \cos \theta_{O W} \\
\gamma=\gamma^{d}+\gamma^{p} \\
\gamma_{S W}=\gamma_{S}+\gamma_{W}-2 \sqrt{\gamma_{S}^{d} \gamma_{W}^{d}}-2 \sqrt{\gamma_{S}^{p} \gamma_{W}^{p}} \\
\gamma_{S O}=\gamma_{S}+\gamma_{O}-2 \sqrt{\gamma_{S}^{d} \gamma_{O}^{d}}-2 \sqrt{\gamma_{S}^{p} \gamma_{O}^{p}}
\end{gathered}
$$

where indices denote S - "solid particle", W - "water" and O - "oil". The values of $\gamma^{\mathrm{d}}$ and $\gamma^{\mathrm{p}}$ for calculations were taken from reference data $[2,7,9]$.

In a number of experiments, a correlation was observed between the value of the edge angle $\theta_{o w}$ of solid emulsifiers and the type, dispersion and stability of the emulsions. Hydrophilic particles with a corner angle $\theta_{o w}<90^{\circ}$ (for example, metal oxides, silica) form direct emulsions or foams, and hydrophobic particles with an angle $\theta_{o w}>90^{\circ}$ (for example, coal, graphite) form inverse emulsions.

The calculated values of free energy of a spherical nanoparticle (in $\mathrm{k}_{\mathrm{B}} \mathrm{T}$ units, $\mathrm{T}=$ $298 \mathrm{~K}$ ) which is located at the interface between water and perfume oil are shown in Figure 2, water and glyceryl trioleate are shown in Figure 3, and Table 1 demonstrates the parameters that were used to calculate this system. Carbon and silica nanoparticles with different degrees of hydrophilicity were chosen as stabilizing impurities. The horizontal axis in Figure 2 is the dimensionless quantity $z$ equal to the distance from the center of the particle to the liquids interface divided by the radius of the particle $(z$, Eq. 1). If the center of the spherical particle is at the interface, then $z=0$, if the particle is completely immersed in the water that is on the left, then $z=-1$, and vice versa, if the particle passes into the oil, then $z=1$. 
The values of the parameters of surface tension coefficients, which were calculated by theoretical formulas, are shown in Table 1.

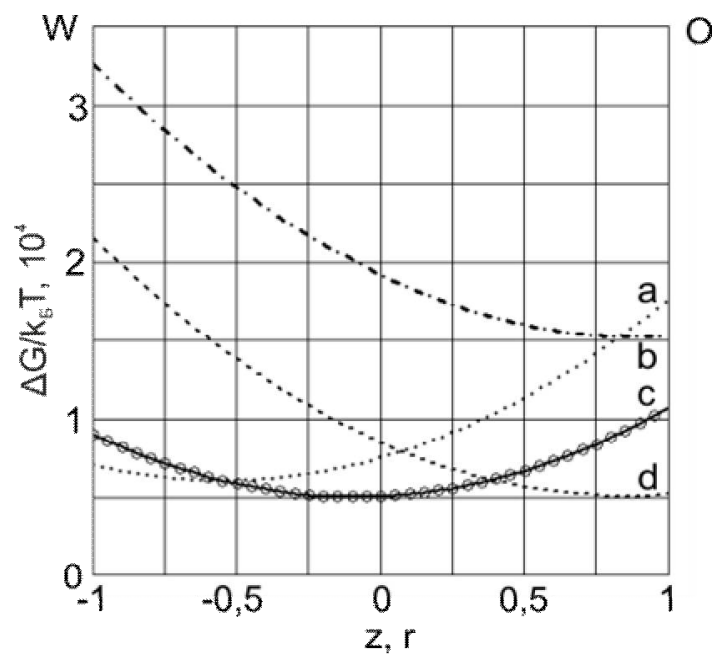

Figure 2. Schematic representation of a quadratic energy well at the perfume oil -water interface $E(z)$ for spherical particles $(\mathrm{R}=12 \mathrm{~nm})$ of hydrophilic silica (a), carbon (b); $\mathbf{5 0 \%}$ hydrophilic (c), and hydrophobic (d) silica ( $W$ is water on the left side, $O$ is perfume oil on the right, and the interface boundary in the middle is $z=0$ ); $z$ is the ratio of the position of the center of the particle to its radius. of the center of the particle to its radius.

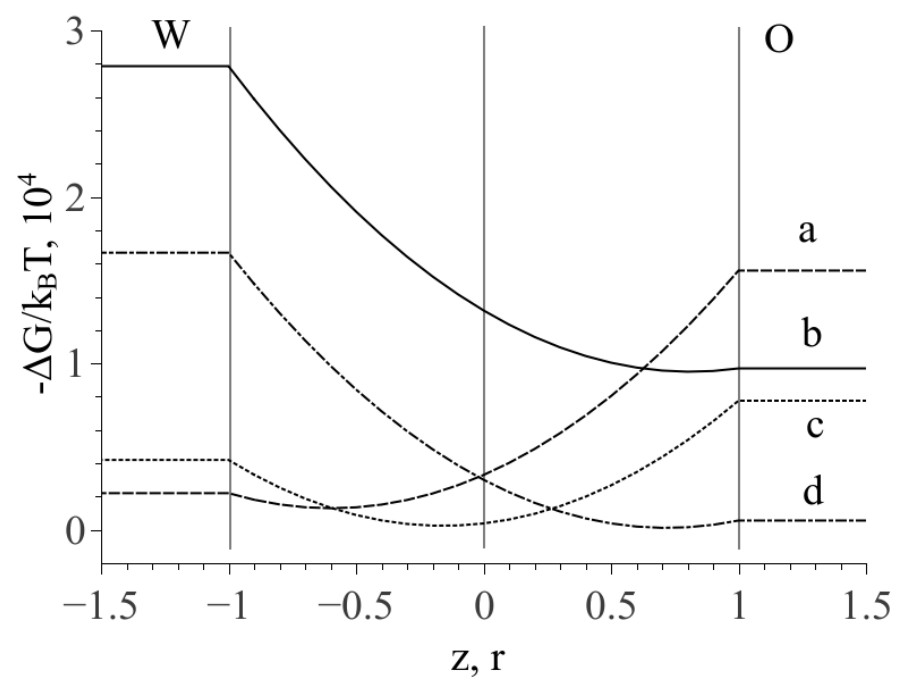

Figure 3. Schematic representation of a quadratic energy well for spherical particles $(R=12 \mathrm{~nm})$ of hydrophilic silica (a), carbon (b);

$50 \%$ hydrophilic (c), and hydrophobic (d) silica at oil (O is glycerin trioleate) - water (W) interface. Other notation is similar to Figure 2. 
Surface tension values at the interface between liquid 1-liquid 2, liquid 1-solid, and liquid 2-solid phases and parameters for the calculations

\begin{tabular}{|c|c|c|c|c|c|c|}
\hline Substance & $\gamma^{\mathrm{d}}, \mathbf{m J} / \mathbf{m}^{2}$ & $\begin{array}{c}\gamma^{p} \\
\mathbf{m} / \mathrm{m}^{2}\end{array}$ & $\underset{\mathrm{mJ} / \mathrm{m}^{2}}{\gamma,}$ & Reference & \multicolumn{2}{|c|}{$\begin{array}{c}\gamma_{\mathrm{x}-\mathrm{y}} \text { calculated, } \\
\mathbf{m J} / \mathbf{m}^{2}\end{array}$} \\
\hline Water & 21.5 & 50.4 & 71.9 & {$[2]$} & - & - \\
\hline $\begin{array}{l}\text { Perfume oil } \\
\text { (O1) }\end{array}$ & $29.9 *$ & $0.3^{*}$ & 30.2 & {$[5]$} & $\gamma_{\mathrm{O} 1-\mathrm{W}}$ & 43.8 \\
\hline $\begin{array}{l}\text { Ethyl esters of } \\
\text { fatty acids of } \\
\text { linseed oil } \\
(\mathrm{O} 2)\end{array}$ & 30.0 & $0.5^{*}$ & 30.5 & [4] & $\gamma_{\mathrm{O} 2-\mathrm{W}}$ & $41.4^{*}$ \\
\hline $\begin{array}{l}\text { Glyceryl } \\
\text { trioleate (O3) }\end{array}$ & 28.1 & 0.0 & 28.1 & [9] & $\gamma_{\mathrm{O} 3-\mathrm{W}}$ & $50.8^{*}$ \\
\hline \multirow[b]{2}{*}{ Carbon } & \multirow[b]{2}{*}{375.0} & \multirow[b]{2}{*}{56.0} & \multirow[b]{2}{*}{431.0} & \multirow[b]{2}{*}{ [7] } & $\gamma_{\mathrm{W}-\mathrm{C}}$ & $63.3^{*}$ \\
\hline & & & & & $\begin{array}{l}\gamma_{\mathrm{O} 1-\mathrm{C}} \\
\gamma_{\mathrm{O} 2-\mathrm{C}} \\
\gamma_{\mathrm{O} 3-\mathrm{C}} \\
\end{array}$ & $\begin{array}{l}23.7 * \\
23.9 * \\
22.0^{*}\end{array}$ \\
\hline \multirow[b]{2}{*}{$\begin{array}{l}\text { Hydrophilic } \\
\text { silica } \\
\mathrm{SiO}_{2}(1)\end{array}$} & \multirow[b]{2}{*}{42.0} & \multirow[b]{2}{*}{34.0} & \multirow[b]{2}{*}{76.0} & \multirow[b]{2}{*}[2]{} & $\gamma_{\mathrm{W}-\mathrm{SiO} 2(1)}$ & $5.0^{*}$ \\
\hline & & & & & $\begin{array}{l}\gamma_{\mathrm{O} 1-\mathrm{SiO} 2(1)} \\
\gamma_{\mathrm{O} 2-\mathrm{SiO} 2(1)} \\
\gamma_{\mathrm{O} 3-\mathrm{SiO} 2(1)}\end{array}$ & $\begin{array}{l}29.1^{*} \\
27.1^{*} \\
35.4^{*}\end{array}$ \\
\hline \multirow{2}{*}{$\begin{array}{l}50 \% \\
\text { hydrophilic } \\
\text { silica } \\
\mathrm{SiO}_{2}(2)\end{array}$} & \multirow[b]{2}{*}{32.0} & \multirow[b]{2}{*}{17.5} & \multirow[b]{2}{*}{49.5} & \multirow[b]{2}{*}[2]{} & $\gamma_{\mathrm{W}-\mathrm{SiO} 2(2)}$ & $9.5 *$ \\
\hline & & & & & $\begin{array}{l}\gamma_{\mathrm{O} 1-\mathrm{SiO} 2(2)} \\
\gamma_{\mathrm{O} 2-\mathrm{SiO} 2(2)} \\
\gamma_{\mathrm{O} 3-\mathrm{SiO} 2(2)}\end{array}$ & $\begin{array}{l}13.4^{*} \\
12.0^{*} \\
17.6^{*}\end{array}$ \\
\hline $\begin{array}{l}\text { Hydrophobic } \\
\text { silica } \\
\mathrm{SiO}_{2}(3)\end{array}$ & 22.0 & 0.9 & 22.9 & {$[2]$} & $\begin{array}{l}\gamma_{\mathrm{W}-\mathrm{SiO} 2(3)} \\
\gamma_{\mathrm{O}-\mathrm{SiO} 2(3)} \\
\gamma_{\mathrm{O} 2-\mathrm{SiO} 2(3)} \\
\gamma_{\mathrm{O} 2-\mathrm{SiO} 2(3)} \\
\end{array}$ & $\begin{array}{c}37.8^{*} \\
0.8 \\
0.7 \\
1.3^{*} \\
\end{array}$ \\
\hline
\end{tabular}

*surface tension values that were calculated by formulas (4-7)

Calculated data of the quadratic energy well for emulsions based on linseed oil fatty acid esters are demonstrated in Figure 4.

It was shown in Figure 4 that a nanoparticle consisting of $50 \%$ hydrophilic silica possesses the best stabilizing properties. The minimum of energy is near the liquids interface with a slight shift toward the water. The stability of a particle is characterized by the energy barrier, from the side of one of the liquids. If the energy barrier is of the same order as the energy of the Brownian motion, the particle will pass without difficulty from the interphase boundary to the liquid volume and stabilization will not occur.

Parameters which were taken to calculate the system consisting of ethyl esters of linseed oil acids with same particles that were selected for the previous calculations, are shown in Table 2. The asterisk shows the theoretically calculated parameters. According to Figure 4, the optimal choices for the material of nanoparticles corresponds to the dependence (c), for which the stabilizing particles at the interface between the phases of the esters of linseed acids - water are formed by $50 \%$ hydrophilic silica. 


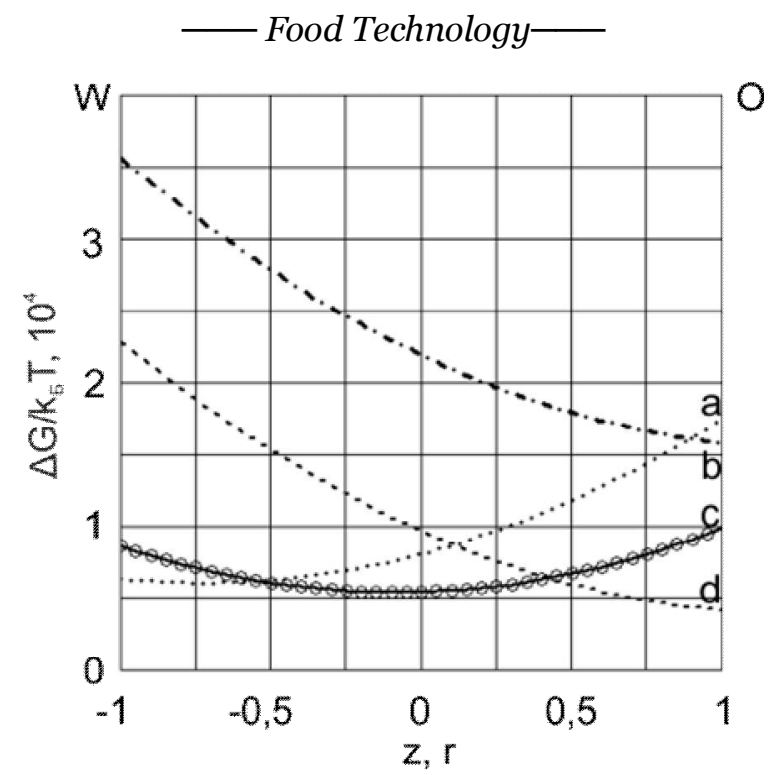

Figure 4. Schematic representation of the quadratic energy well $E(z)$ at the linseed acids esters (O) - water (W) interface for spherical particles of hydrophilic silica (a), carbon particles (b); $50 \%$ hydrophilic (c) and hydrophobic (d) silica.

Due to the fact that the parameters of the esters of acids of linseed oil and perfume oil are quite similar, the absence of qualitative changes in the graphs have occurred. Nanoparticles of $50 \%$ hydrophilic silica were stabilized near the phase interface with a small displacement of the particle toward the water and had the same. best stabilizing properties.

Let us analyze the obtained data for the paraffin. The surface tension of solid-air $\left(\mathrm{mJ} / \mathrm{m}^{2}\right)$ for non-polar hydrophobic paraffin is $\gamma_{S}=\gamma_{S}^{d}=23.9, \gamma_{S}^{p}=0$ [10]. We will change the system, choosing triglycerides of carboxylic acids $\mathrm{C}_{4}$ and $\mathrm{C}_{6}$ as the oil. The experimental values of the parameters are given in [10]; calculated and reference data are summarized in Table 2. Quadratic energy wells for triglycerides of carboxylic acids are shown in Figure 5. In this case the oil, which the emulsion consists, was varied. Paraffin particles best stabilize the Glyceryl tributyrate. As can be seen from Figure 5, the particles stabilize water-in-oil emulsion, because they show a high hydrophobicity.

Table 2

Surface tension $\left(\mathrm{mJ} / \mathrm{m}^{2}\right)$ for triglycerides on the phases' interface with air [10] and the calculated values of surface tension at the oil-water $\left(\gamma_{O W}\right)$, paraffin-water $\left(\gamma_{S W}\right)$

and paraffin-oil $\left(\gamma_{S O}\right)$ systems

\begin{tabular}{|c|c|c|c|c|c|c|}
\hline Triglyceride (oil) & $\gamma_{O}^{p}$ & $\gamma_{O}^{d}$ & $\gamma_{O W}$ & $\gamma_{O W}$ & $\gamma_{S W}$ & $\gamma_{S O}$ \\
\hline Tributyrate $\left(\mathrm{C}_{4}\right)$ & 27.7 & 2.8 & 30.5 & $29.8 *$ & $26.4 *$ & $2.9 *$ \\
\hline Tricaprylate $\left(\mathrm{C}_{6}\right)$ & 25.9 & 3.3 & 29.2 & $28.1 *$ & $26.4 *$ & $3.3 *$ \\
\hline
\end{tabular}

*Values of surface tension calculated by formulas (4-7). 


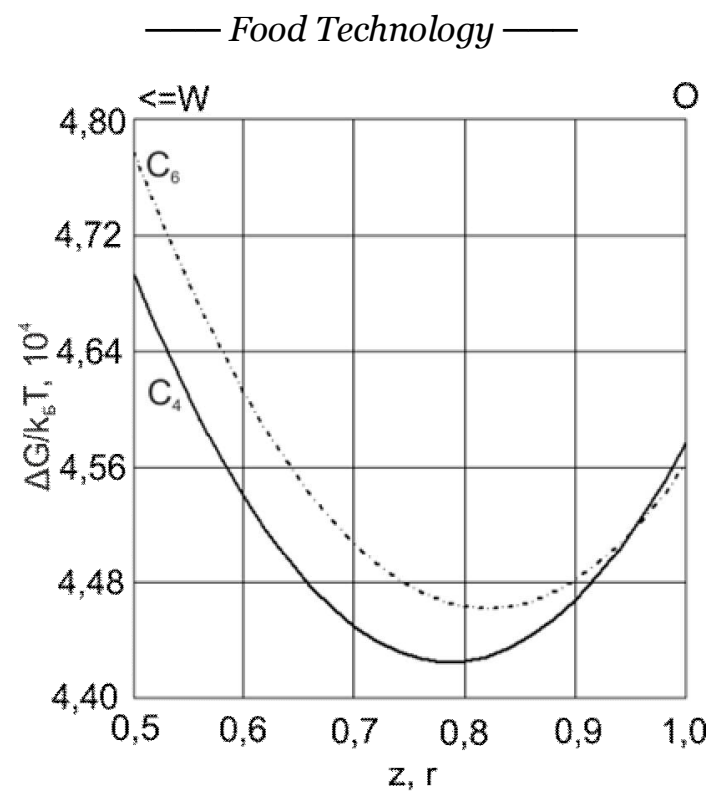

Figure 5. Quadratic energy well for nonpolar hydrophobic paraffin nanoparticles, which stabilizing emulsions of triglycerides of carboxylic acids: tributyrate $\left(C_{4}\right)$ and tricaprylate $\left(C_{6}\right)$. Water is the left edge, triglycerides is the right edge, the liquids interface is in the middle $(z=0)$.

Table 3

Energy barriers for the transfer of the selected emulsifier particle to the bulk phase of the oil ( $\left.\Delta E_{S O}\right)$ and to the water $\left(\Delta E_{S W}\right)$, in $\mathbf{k}_{\mathbf{B}} \mathbf{T}$ units*

\begin{tabular}{|l|c|c|c|c|}
\hline Triglyceride (oil) & $\mathbf{z}_{\mathbf{m}}$ & $\Delta E_{S O}$ & $\Delta E_{S W}$ & $\Delta E_{\boldsymbol{m i n}}$ \\
\hline \multicolumn{5}{|c|}{ 50\% hydrophilic silica } \\
\hline Glycerol trioleate & $\mathbf{- 0 . 2}$ & $\mathbf{7 5 0}$ & $\mathbf{4 0 0}$ & $\mathbf{4 0 0}$ \\
\hline Perfume oil & $\mathbf{- 0 . 0 9}$ & $\mathbf{5 6 9 0}$ & $\mathbf{4 0 1 0}$ & $\mathbf{4 0 1 0}$ \\
\hline Linseed acids esters & $\mathbf{- 0 . 0 8}$ & $\mathbf{3 9 2 0}$ & $\mathbf{2 8 3 0}$ & $\mathbf{2 8 3 0}$ \\
\hline \multicolumn{5}{|c|}{ Carbon } \\
\hline Glycerol trioleate & 0.80 & 200 & 1840 & 200 \\
\hline Perfume oil & 0.90 & 40 & 1750 & 40 \\
\hline Linseed acids esters & 1.29 & - & - & - \\
\hline \multicolumn{5}{|c|}{ Paraffin } \\
\hline Tributyrate $\left(\mathrm{C}_{4}\right)$ & 0.79 & 150 & 1040 & 150 \\
\hline Tricaprylate $\left(\mathrm{C}_{6}\right)$ & 0.81 & 100 & 1020 & 100 \\
\hline
\end{tabular}

*optimal compositions from the point of view of the Pickering stabilization are selected in bold

In connection with the fact that the energy wells are parabolas, information on the magnitude of the barriers and the position of the minimum can be represented in the form of Table 3. Energy barrier values $\Delta E_{S O}$ and $\Delta E_{S W}$ are presented in Table 3 in $\mathrm{k}_{\mathrm{B}} \mathrm{T}$ units when the particle is moved from the minimum with the $z_{m}$ coordinate into water $(z=-1)$ and 
into oil $(z=1)$. Thus, a sufficiently strong stabilizing effect is generally observed for the triglycerides which chosen by us for model calculations.

Stabilizing particles, which corresponds to optimal selection of the nanoparticle material, are consisting of 50\% hydrophilic silica. The best energy well parameters of this solid emulsifier make about 2830 (in linseed oil and in their ethers), 4010 (perfume oil), 400 (triolein) and $150 k_{B} T$ (tributyrin). In our opinion, $\Delta E_{\text {min }}$ can be general parameter of nanoparticles ability to be stabilized at $\mathrm{O} / \mathrm{W}$ interface of an emulsion (Table 3 ). The more $\Delta E_{\min }$ value the higher is the stability. Therefore, the best nanomaterial is again $50 \%$ hydrophilic silica and perfume oil / water interface with $50 \%$ hydrophilic silica represents the most stable emulsion.

\section{Conclusions}

The principal possibility of theoretical prediction of the conditions for the stabilization of food and cosmetic emulsions by the Pickering effect is shown. The parameters of the system are calculated which indicate the most effective composition of the emulsions: the position of the particle relative to the interface of the liquids, the energy barriers corresponding to the transfer of the particle from the energy minimum position, if this energy minimum is in the boundary layer between oil and water. One of the conditions for the stabilization of emulsions by solid nanoparticles is the existence of an energy stabilizing "well" due to the difference in the surface tension of the interacting phases.

The appearance of the Pickering effect is confirmed by calculations for a number of model oil-water systems, consisting of linseed oil and its ethyl esters, perfume oil, triglycerides of carboxylic $\mathrm{C}_{4}, \mathrm{C}_{6}$ and oleic acids, and water. In the case of solid nanoparticles, paraffin, activated carbon, hydrophilic, hydrophobic silica and 50\% hydrophobized silica were chosen.

The parameters of the systems that indicate the type of stable emulsion are calculated (the positions of the particle relative to the interface of the liquids, the value of the energy barriers which corresponding to the transfer of the particle from the energy minimum position [if this minimum lies in the boundary layer] into the bulk oil or water phase) and their most effective compositions were chosen.

Stabilizing particles, which corresponds to optimal selection of the nanoparticle material, are consisting of $50 \%$ hydrophilic silica. The best energy well parameters of this solid emulsifier make about 2830 (in linseed oil and in their ethers), 4010 (perfume oil), 400 (triolein) and $150 k_{B} T$ (tributyrin). In our opinion, $\Delta E_{\min }$ can be general parameter of nanoparticles ability to be stabilized at $\mathrm{O} / \mathrm{W}$ interface of an emulsion. The more $\Delta E_{\text {min }}$ value the higher is the stability. Therefore, the best nanomaterial is again $50 \%$ hydrophilic silica and perfume oil / water interface with $50 \%$ hydrophilic silica represents the most stable emulsion.

The chosen systems modelled for stabilization of emulsions by nanoparticles are close to real food and cosmetic objects and were limited first of all experimental or theoretical data available. For the best of our knowledge, 50\% hydrophilic silica exhibits the optimal parameters and the further work on experimental confirmation of effects considered is to be planned. The prospective direction of the further researches could be the study of other oxides with partially hydrophilic surface. 


\section{References}

1. Bon S. A. F. (2014), Particle-Stabilized Emulsions and Colloids: Formation and Applications. Chapter 1, RSC Publishing.

2. Binks B. P., Clint J. H. (2002), Solid Wettability from Surface Energy Components: Relevance to Pickering Emulsions, Langmuir, 18 (4), pp. 1270-1273.

3. Dickinson E. (2012), Use of nanoparticles and microparticles in the formation and stabilization of food emulsions, Trends in Food Science \& Technology, 24 (1), pp. 4 12.

4. Matthäus B., Özcan M. M. (2017), Fatty Acid Composition, Tocopherol and Sterol Contents in Linseed (Linum usitatissimum L.) Varieties, Iran. J. Chem. Chem. Eng., 36 (3), pp. 147-152.

5. Hashemi S. M. B., Khaneghah A. M., Sant'Ana A. de S. (2017), Essential Oils in Food Processing: Chemistry, Safety and Applications, John Wiley \& Sons.

6. Barel A. O., Paye M., Maibach H. I. (2014), Handbook of Cosmetic Science and Technology, CRC Press.

7. Kwok S. C. H., Wang J., Chu P. K. (2005), Surface energy, wettability, and blood compatibility phosphorus doped diamond-like carbon films, Diam. Relat. Mater., 14 (1), pp. 78-85.

8. (2017), PTC Mathcad | PTC, available at: https://www.ptc.com/en/engineering-mathsoftware/mathcad.

9. XU T. (2017), Food Oil Surface and Interfacial Tension as Affected by Composition, Time and Temperature, Dissertation for the degree of doctor of philosophy in Food Science, University of California, Davis.

10. Saito M., Otani M., Yabe A. (1985), Work of Adhesion of Oily Dirt and Correlation with Washability, Text. Res. J., 55 (3), pp. 157-164. 